\title{
Long-term lithium treatment in bipolar disorder is associated with longer leukocyte telomeres
}

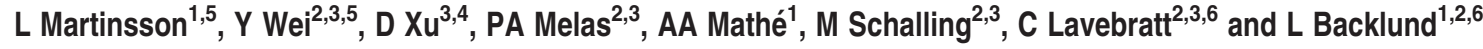

Telomere shortening is a hallmark of aging and has been associated with oxidative stress, inflammation and chronic somatic, as well as psychiatric disorders, including schizophrenia and depression. Additionally, antidepressants have been found to protect against telomere shortening. However, pharmacological telomere studies are lacking in bipolar disorder (BD). Therefore, the objective of this study was to explore telomere length (TL) in patients with BD in the context of lithium treatment. We determined TL by quantitative real-time PCR using peripheral blood leukocytes. Participants were outpatients diagnosed with BD type 1 or 2 $(n=256)$ and healthy controls $(n=139)$. Retrospective case-control and case-case study designs were applied. Lithium response (LiR) was scored using the Alda-Scale. Lithium-treated BD patients overall, as well as those on lithium monotherapy, had $35 \%$ longer telomeres compared with controls $\left(P<0.0005\right.$, partial $\left.\eta^{2}=0.13\right)$. TL correlated positively with lithium treatment duration of $>30$ months $\left(P=0.031, R^{2}=0.13\right)$ and was negatively associated with increasing number of depressive episodes $(P<0.007)$. BD patients responding well to lithium treatment had longer telomeres than those not responding well. This is the first study to report a positive effect of long-term lithium treatment on TL. Importantly, longer TL was also associated with a better LiR in BD patients. These data suggest that lithium exerts a protective effect against telomere shortening especially when therapeutically efficacious. We hypothesize that induction of telomerase activity may be involved in LiR in BD.

Translational Psychiatry (2013) 3, e261; doi:10.1038/tp.2013.37; published online 21 May 2013

\section{Introduction}

Bipolar disorder (BD) is a severe and chronic disorder affecting about $1-2 \%$ of the population causing immense suffering and excessive costs. ${ }^{1,2}$ BD type 1 is characterized by alternating episodes of depression and mania, whereas BD type 2 is characterized by depressions and hypomanias. ${ }^{3}$ Rapid cycling (RC) is a severe subtype of BD defined as four or more bipolar episodes within a year, often difficult to stabilize with treatment. It has been suggested that RC should be considered a separate diagnostic entity apart from the BD, but this topic is still under discussion. ${ }^{4}$

Lithium is the first-line mood stabilizer in BD and has a unique role in suicide prevention. ${ }^{5}$ However, $40-50 \%$ of bipolar patients relapse within 2 years of lithium treatment. ${ }^{6}$ Lithium response (LiR) clusters in families to a large extent, but it is difficult to identify these responders a priori among patients because of the lack of predictive biomarkers. ${ }^{7}$ Lithium is thought to stabilize neuronal activities and to promote neural plasticity and neuroprotection. ${ }^{8}$ Lithium has probably several additional modes of action that are important for its therapeutic effects, but these mechanisms remain poorly understood.

Telomeres are DNA nucleoprotein complexes forming the ends of linear eukaryotic chromosomes, which protect the latter from natural cellular erosion and fusion with each other. However, telomeres erode progressively with each cell division, partly because of the end replication problem and also because of oxidative stress, finally signaling cellular senescence and apoptosis. ${ }^{9}$ The efficiency of TL maintenance has been reported to be affected by sex, ${ }^{10}$ genetic variation (for example, in the TERT gene encoding the telomerase reverse transcriptase) and environmental factors (for example, cigarette smoking). ${ }^{11}$ Aging and somatic diseases, such as cancer, cardiovascular diseases, diabetes, dementia and cognitive decline, have all been associated with telomere shortening. ${ }^{12}$ Furthermore, early life stress, depression and hypocortisolemia have also been negatively correlated with $\mathrm{TL}$, suggesting that shorter telomeres might be a cumulative measure of stress in life. ${ }^{13}$ In contrast to the above-mentioned factors that lead to TL shortening, factors like physical activity and effective pharmacological treatment have been associated with increased TL and telomerase activity, respectively. ${ }^{14,15}$

In psychiatric disorders, shorter leukocyte TL (LTL) has been associated with short sleep duration, phobic anxiety, schizophrenia and depression. ${ }^{12,16,17}$ Previous studies also suggest that the number of depressive episodes is associated with shorter $\mathrm{LTL}^{18}$ and that antidepressant pharmacological

\footnotetext{
${ }^{1}$ Department of Clinical Neuroscience, Centre for Psychiatric Research and Education, Karolinska Institutet, Clinic for Affective Disorders, Karolinska University Hospital, Stockholm, Sweden; ${ }^{2}$ Neurogenetics Unit, Department of Molecular Medicine and Surgery, Karolinska Institutet, Stockholm, Sweden; ${ }^{3}$ Center for Molecular Medicine, Karolinska University Hospital, Stockholm, Sweden and ${ }^{4}$ Division of Hematology, Department of Medicine, Karolinska University Hospital and Karolinska Institutet, Solna, Sweden

Correspondence: Dr L Martinsson, Department of Clinical Neuroscience, Centre for Psychiatric Research and Education, Karolinska Institutet, The Clinic for Affective Disorders, Karolinska University Hospital, Stockholm S-141 86, Sweden.

E-mail: lina.martinsson@ki.se

${ }^{5}$ The first two authors contributed equally to this work.

${ }^{6}$ The last two authors contributed equally to this work.

Keywords: Alda-Scale; biomarker; lithium response; number of episodes; rapid cycling; telomerase

Received 11 February 2013; revised 27 March 2013; accepted 8 April 2013
} 
treatment protects against telomere shortening. ${ }^{15,17}$ In addition, shorter LTL has been associated with poor response to medical treatment in schizophrenia. ${ }^{19}$ To our knowledge, only one study with regard to BD has been conducted, which using a small group of patients $(n=28)$ found an association of $\mathrm{BD}$ and number of depressive episodes with shorter LTL. ${ }^{20}$ However, despite neuroprotective action of lithium, ${ }^{8}$ there has been no study to examine $T L$ in $B D$ patients with regard to lithium treatment or LiR. Thus, in the present study, we tested whether (i) lithium treatment in BD patients may affect LTL, (ii) LTL in BD patients may associate with lithium responsiveness and (iii) number of depressive episodes, as well as RC, may be linked to $L T L$.

\section{Materials and methods}

Subjects. Patients $(n=789)$ with clinical diagnosis of $\mathrm{BD}$ were consecutively recruited from the Unit of Affective Disorders at Psychiatry Southwest, Huddinge Hospital, Stockholm. Life-time manic and depressive symptoms were assessed by a psychiatrist specialized in BD or by a trained psychiatric nurse using the modules for mania and depression in the Schedules for Clinical Assessment in Neuropsychiatry as previously described. ${ }^{21}$ On the basis of these assessments, patients were considered as fulfilling the diagnostic criteria for BD type 1, 2 or not otherwise specified. The symptoms as well as the number of manias and depressive episodes, RC and mixed episodes were assessed, including the age of onset of mania and depression. Two cohorts were selected according to predefined diagnostic and treatment response criteria. The first one (set I) consisted of patients with excellent LiR $(n=76)$ and sex- and age-matched patients with none or partial LiR (non-LiR, $n=76$ ), all with therapeutic serum concentration $\left(0.5-0.9 \mathrm{mmoll}^{-1}\right)$ during a period of at least 3 months before DNA sampling. The second set (set II) consisted of RC patients $(n=98)$ and sex- and age-matched patients without RC (non-RC, $n=98$ ). From these four groups, DNA was available from 65, 65, 97 and 94 individuals, respectively. LiR was measured according to the yearly reduction of numbers and/or severity of episodes after the beginning of lithium treatment, controlled for the duration of lithium treatment (defined as months on treatment with serum concentration of $0.5-0.9 \mathrm{mmoll}^{-1}$ ), the age of onset of $\mathrm{BD}$, the number of years with $B D$ before treatment, the duration of lithium treatment before DNA sampling and the presence of other mood stabilizers, antidepressants, neuroleptics or sleep medication according to the Alda-Scale., 22 In previous studies, LiRs were those who scored $\geqslant 7$ and non-LiRs were those who scored $\leqslant 6$ (range $0-10$ points). In order to achieve two well-defined groups according to LiR, patients who scored 6 points were excluded. Thus, LiR were those who scored $\geqslant 7$ and non-LiR were those who scored $\leqslant 5$. No other thresholds for LiR or non-LiR were analyzed. The BD patients on therapeutic lithium serum concentration during at least 3 months were stratified into two subgroups: lithium monotherapy and lithium plus add-on medications. Healthy non-obese controls were selected from two longitudinal population-based studies in Stockholm, Sweden: the Diabetes Prevention Program ${ }^{23}$ and the PART study of mental health. ${ }^{24}$
The study was approved by the Regional Ethical Review Board in Stockholm, Sweden, in accordance with the Helsinki Declaration of 1975 . For patients, both written and verbal informed consent was obtained at a visit with a specialized psychiatric nurse during euthymic state. For controls, verbal or written informed consent was obtained according to procedures approved by the ethical committee. All individuals had full capacity to consent. The procedure was documented in the research protocol, fulfilling the Swedish legal requirements.

LTL determination by quantitative real-time PCR. Peripheral blood samples were collected in EDTA tubes and DNA was extracted using an SDS-urea and phenol/ chloroform/isoamylalcohol procedure, followed by purification using illustra NAP-5 columns (GE Healthcare, Buckinghamshire, UK). Relative LTL was determined according to Cawthon et al. ${ }^{25}$ and Kananen et al. ${ }^{26}$ In brief, DNA samples $(4 \mathrm{ng})$ were processed in triplicates both for the telomere (Tel) and the single-copy gene (hemoglobin- $\beta$, Hgb) PCR, within the same 384 -well plate using Platinum ${ }^{\circledR}$ SYBR $^{\circledR}$ Green qPCR SuperMix-UDG (Invitrogen, by Life Technologies, Carlsbad, CA, USA) according to the manufacturer's instructions. The primers $(10 \mathrm{~nm})$ for the telomere PCR were Tel1 (5'-GGTTTTTGAGGGTGAGGGTGAGGGTGAGGGT GAGGGT-3') and Tel2 (5'-TCCCGACTATCCCTATCCCTA TCCCTATCCCTATCCCTA-3') and for the Hgb PCR were Hgb1 (forward primer; 5'-GCTTCTGACACAACTGTGTTC ACTAGC-3') and Hgb2 (reverse primer; 5'-CACCAACTTC ATCCACGTTCACC-3'). The PCR temperature conditions for Tel and $\mathrm{Hgb}$ were $95^{\circ} \mathrm{C}$ for 2 min followed by 40 cycles of $95^{\circ} \mathrm{C}$ for $15 \mathrm{~s}$ and $62^{\circ} \mathrm{C}$ for $1 \mathrm{~min}$, including a dissociation curve to indicate specificity. The procedures were carried out on an ABI PRISM 7900 HT qPCR instrument (Applied Biosystems, Foster City, CA, USA). DNA samples with a standard deviation of $>0.4$ between triplicates or a Ct value of $>36$ were omitted from the analyses $(n=13)$. The relative gene expression was calculated according to the comparative $2^{-\Delta \Delta \mathrm{Ct}}$ method, where $\Delta \Delta \mathrm{Ct}=\Delta \mathrm{Ct}_{\text {sample }}-\Delta \mathrm{Ct}_{\text {calibrator }}$ sample and $\Delta \mathrm{Ct}_{\text {sample }}=\mathrm{Ct}_{\mathrm{Tel}}-\mathrm{Ct}_{\mathrm{Hgb}}$. Samples from $\mathrm{BD}$ patients and healthy controls were assayed in the same 384-well plate. Inter-plate calibration was performed by normalizing $2^{-\Delta \Delta C t}$ of each sample to the average of three control samples. A dilution series of a synthetic oligomer for both Tel (TTAGGGx14: 107, 10.7, 1.07, 0.107, 0.0107 and $0.00107 \mathrm{pg})$ and Hgb (119-mer: 15, 1.5, 0.15, 0.015, 0.0015 and $0.00015 \mathrm{pg}$ ) were included in each plate to create reaction-specific standard curves. The mean of the correlation coefficient of the standard curves was $>0.99$ for both the Tel and the Hgb reaction.

Statistical analyses. Differences in clinical characteristics between study groups were assessed using Pearson's chisquare test or Mann-Whitney U-test. LTL dependence on sex and age was determined using analysis of covariance (ANCOVA). Correlation between LTL and age was determined using nonparametric Spearman's correlation test. Likewise, partial correlation between LTL and duration of lithium treatment, controlled for age and gender, was also determined using nonparametric Spearman's correlation test. Difference in duration of lithium treatment between LiR 
and non-LiR groups was determined using nonparametric Mann-Whitney U-test. Dependence of LTL on duration of lithium treatment and on number of depressive episodes was assessed using linear regression while adjusting for age and sex; partial Cohens eta square $\left(\eta^{2}\right)$ indicates effect size (proportion variance explained) similar to $R^{2}$ in ANCOVA; $\eta^{2}=0.01$ small; $\eta^{2}=0.06$ moderate; $\eta^{2}=0.14$ large effect. Residuals from linear regression analyses were controlled for normality. Dependence of LTL on the dichotomous variables $\mathrm{RC}$ and response to lithium treatment was tested using ANCOVA adjusted for age and sex. Similarly, difference in LTL between BD patients on lithium and healthy controls was assessed in the sex- and age-matched sample (age range: 33-77 years) using ANCOVA, while adjusting for sex and age, and was then verified using nonparametric Mann-Whitney $U$-tests. A $P$-value of $<0.05$ was regarded as statistically significant. The analyses were performed using IBM SPSS Statistics v. 20 (IBM Corporation, Armonk, NY, USA).

\section{Results}

The characteristics of the study groups are shown in Table 1. LTL correlated negatively with age $(F=42.6, P<0.0005$, Spearman's $\rho=-0.28)$, but was independent of sex $(P>0.05)$ in patients and controls. There was no age $\times$ sex interaction on LTL $(P>0.15)$.

LTL and lithium treatment duration in BD. As lithium has a neuroprotective effect, ${ }^{8}$ we tested whether lithium exposure influenced LTL. This was assessed in Set I comprising of ageand sex-matched LiR and non-LiR BD patients with a duration of lithium treatment that was for LiR: 52 (21,172), 3-468 months, and for non-LiR: 46 (21,113), 3-360 months (numbers indicate: median (25th,75th percentile) range). Lithium duration did not differ between the LiR and the nonLiR groups ( $P=0.36$, Mann-Whitney U-tests). LTL correlated positively with lithium treatment duration given exposure for more than 30 months ( $P=0.044$, Spearman's $\rho=0.24)$ and was also positively dependent on lithium treatment duration as determined by linear regression $(n=72$; lithium treatment duration: standardized (std) $\beta=0.29, t=2.2, P=0.031$; age: std $\beta=-0.41, t=3.1, P=0.003, R^{2}=0.13$; Figure 1). Sex had no effect on this association. In the whole of Set I, with a lithium duration range of 3-468 months, LTL was not significantly dependent on lithium treatment duration ( $n=121$; lithium treatment duration: std $\beta=0.13, t=1.2$, $P=0.22$; age: std $\beta=-0.48, t=4.5, P<0.0005)$.

LTL and number of episodes. There was a tendency for shorter LTL in the RC cases compared with the age- and sex-matched non-RC cases (Set II), which was, however, not statistically significant $(\Delta=6 \%, \mathrm{~F}=2.16, P=0.14$, ANCOVA

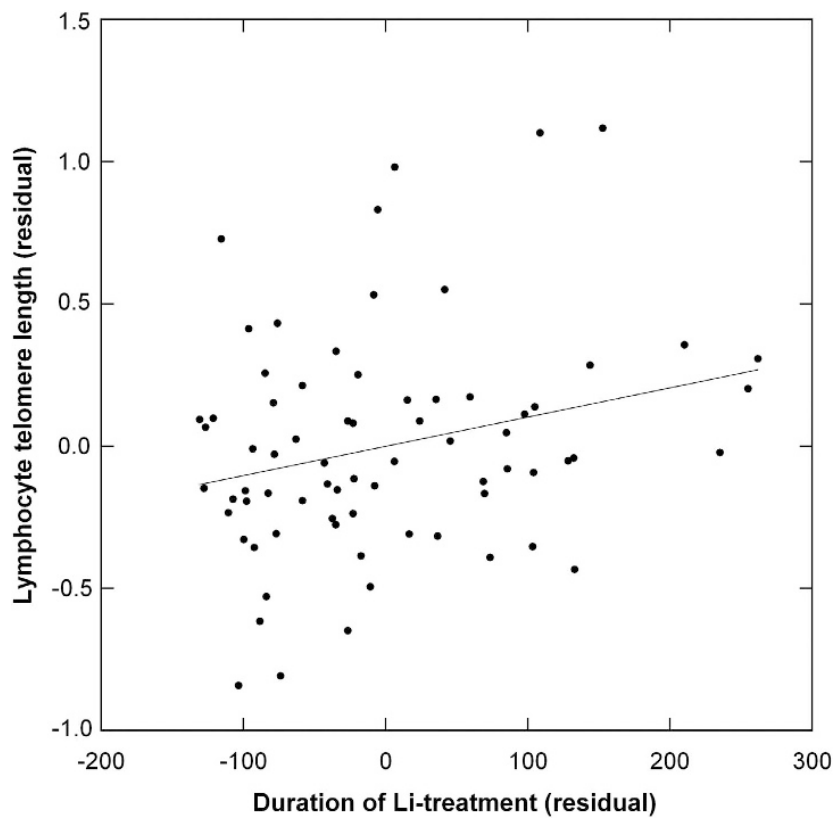

Figure 1 Lymphocyte telomere length (LTL) was positively dependent on duration of lithium treatment among those exposed for more than 30 months $(n=71, P=0.032$, Spearman's $\rho=0.24)$. Axes represent unstandardized residuals of regressing LTL on age and sex, and regressing duration of lithium treatment on age and sex.

Table 1 Clinical characteristics of the study groups

\begin{tabular}{|c|c|c|c|c|c|c|c|c|c|}
\hline Group & $\mathrm{n}^{\mathrm{a}}$ & $\begin{array}{c}\text { Males } \\
(\%)\end{array}$ & Age (years) ${ }^{\mathrm{a}}$ & $\begin{array}{c}\text { Disease } \\
\text { duration (years) }{ }^{\mathrm{a}} \wedge\end{array}$ & $\begin{array}{c}\text { Mixed } \\
\text { episode } \\
(\% \text { patients })\end{array}$ & $\begin{array}{c}\text { Mania or } \\
\text { hypomania } \\
\left(\mathrm{n}_{\text {episodes }}\right)^{\mathrm{a}, \mathrm{b}}\end{array}$ & $\begin{array}{l}\text { Depression } \\
\left(\mathrm{n}_{\text {episodes }}\right)\end{array}$ & $\begin{array}{c}R C \\
\left(\%_{\text {patients }}\right)\end{array}$ & $\begin{array}{c}\text { BD2 } \\
(\% \text { patients })\end{array}$ \\
\hline \multicolumn{10}{|l|}{ Set I } \\
\hline Lithium responder & 65 & 44.6 & $50(36,63)$ 21-89 & $28(15-40) 4-72$ & $48.4^{*}$ & $3(2,6)^{*}$ & $6(5,6)^{\star \star \star}$ & $21.5^{\star}$ & $8.6^{*}$ \\
\hline $\begin{array}{l}\text { Non-lithium } \\
\text { responder }\end{array}$ & 65 & 45.3 & $52(44,62)$ 22-84 & $30(22,38) 1-58$ & 32.3 & $6(3,6)$ & $6(6,6)$ & 43.8 & 17.9 \\
\hline \multicolumn{10}{|l|}{ Set II } \\
\hline Rapid cycling & 97 & 40.4 & $49(35,61) 23-80$ & $22(13,39) 1-56$ & 46.4 & $6(3,6)^{\star \star}$ & $6(6,6)^{\star \star \star}$ & 100 & $0^{*}$ \\
\hline No rapid cycling & 94 & 46.4 & $52(40,62) 21-78$ & $26(15,36) 6-62$ & 31.9 & $4(2,6)$ & $6(3,6)$ & 0 & 23.7 \\
\hline \multicolumn{10}{|l|}{ Combined } \\
\hline Bipolar disorder ${ }^{\mathrm{C}}$ & 256 & 43.0 & $50(38,62) 21-89^{\star *}$ & $27(15,37) 1-72$ & 39.6 & $5(2,6)$ & $6(4,6)$ & 43.8 & 12.8 \\
\hline Bipolar disorder ${ }^{d}$ & 207 & 45.5 & $53(43,63) 33-77$ & $29(20,38) 1-58$ & 39.9 & $5(3,6)$ & $6(5,6)$ & 42.7 & 12.6 \\
\hline
\end{tabular}

Abbreviations: BD, bipolar disorder; RC, rapid cycling.

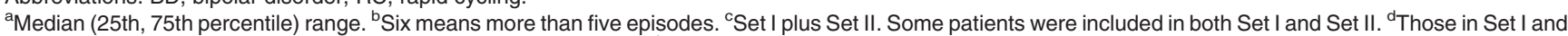
Set II with therapeutic serum lithium concentration $\left(0.5-0.9 \mathrm{mmolI}^{-1}\right)$ in at least a period of 3 months before DNA sampling, with same age range as controls. Some patients were included in both Set I and Set II. ${ }^{\star} P<0.05 ;{ }^{* \star} P<0.01 ;{ }^{* \star \star} P<0.001$ compared with the 'No' category (Set I and Set II), compared with controls (combined sample) using Pearson's $\chi^{2}$ test or Mann-Whitney U-test. 
adjusted for age $(P<0.0005)$ and sex $(P=0.57) ; P=0.093$, Mann-Whitney U-tests; Table 1 and Figure 2a). When assessing the combined group of $\mathrm{RC}$ and non-RC cases, there was a significant effect of the number of depressive episodes on LTL, with an effect size similar to that of age (depressive episodes: std $\beta=-0.20, P=0.007$; age: std $\beta=-0.26, \quad P<0.0005, \quad R^{2}=0.14 ;$ Figure $\left.2 b\right)$. The $\mathrm{LTL}$ (relative quantity) marginal mean was reduced 0.075 units per depressive event after adjusting for age and sex assuming linear model. This dependence on number of depressive episodes was stronger in males (std $\beta=-0.32$, $P=0.006$ ) than in females (std $\beta=-0.11, P=0.29$ ). As there was an effect of the number of depressive episodes on LTL, the LTL dependence on lithium treatment duration was corrected for the number of depressive episodes. However, the number of depressive episodes was not found to influence the association between LTL and lithium treatment duration $(P=0.62)$. For manic/hypomanic episodes, no dependence between LTL and number of episodes was found.

LTL and response to lithium. LTL was also assessed for difference between LiR and non-LiR groups in Set I. LTL was $10 \%$ longer in the LiR patients compared with the non-LiR patients after adjusting for all the parameters here suggested to influence $L T L$ ( $L i R$ vs non-LiR: $F=4.31, P=0.047$, partial $\eta^{2}=0.13$; age: $\mathrm{F}=10.8, P=0.003$; number of depressive episodes: $\mathrm{F}=2.29, P=0.014$; lithium treatment duration: $\mathrm{F}=1.41, \quad P=0.15 ;$ sex: $\mathrm{F}=0.61, \quad P=0.44$ : ANCOVA; Figure 3). As shown here, increasing number of depressive episodes had a shortening effect on LTL also in Set I. The absence of a significant effect of lithium treatment duration on LTL in the above-mentioned model was due to the inclusion in the analysis of those individuals with a short lithium treatment duration.
LTL in BD compared with healthy controls. The mean value of LTL was $34.5 \%$ higher in BD cases $(n=202)$ compared with healthy controls $(n=135 ; \quad \mathrm{F}=49.9$, $P<0.0005$, partial $\eta^{2}=0.13$, ANCOVA adjusted for sex $(P=0.10) \quad$ and age $\left(P<0.0005\right.$, partial $\left.\eta^{2}=0.043\right)$; $P<0.0005$, Mann-Whitney $U$-tests; Figure 3). Not only LiR,

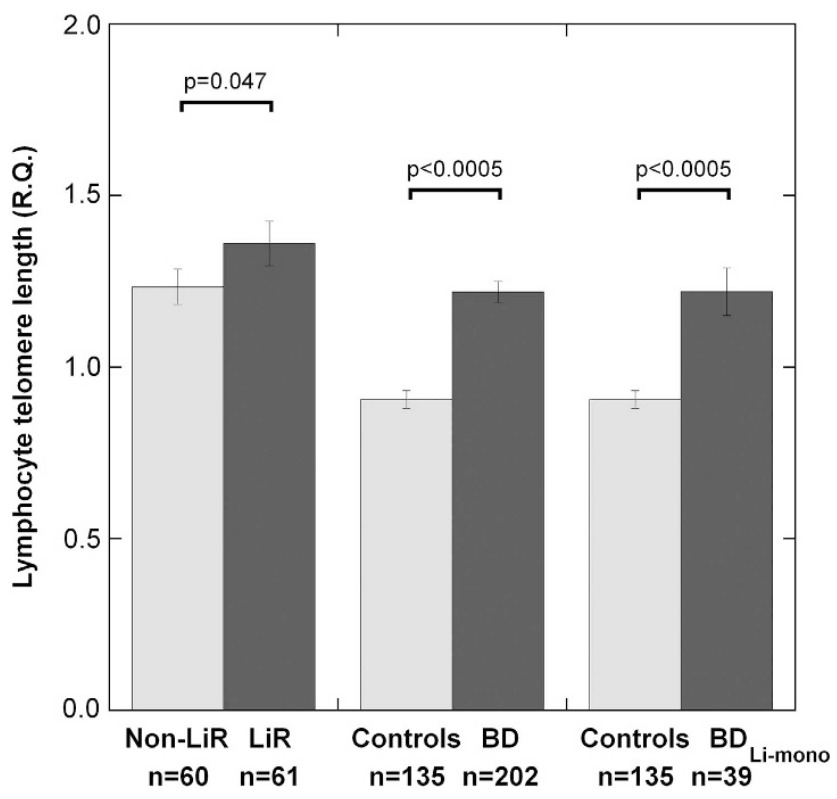

Figure 3 Lymphocyte telomere length (LTL) was increased in those responding well to lithium treatment compared with those not responding well to lithium treatment. LTL was increased in bipolar disorder (BD) patients, also in those on lithium monotherapy $\left(\mathrm{BD}_{\mathrm{Li}-\mathrm{mono}}\right)$, compared with healthy controls with similar sex and age distribution. Not only the lithium responders, but also the non-lithium responders had longer LTL compared with healthy controls $(P<0.0005)$. The $y$ axis represents LTL relative quantity $(R Q)$ values and patients in LiR and non-LiR groups are matched for age and sex. Bars indicate the mean and error bars indicate standard error of the mean.
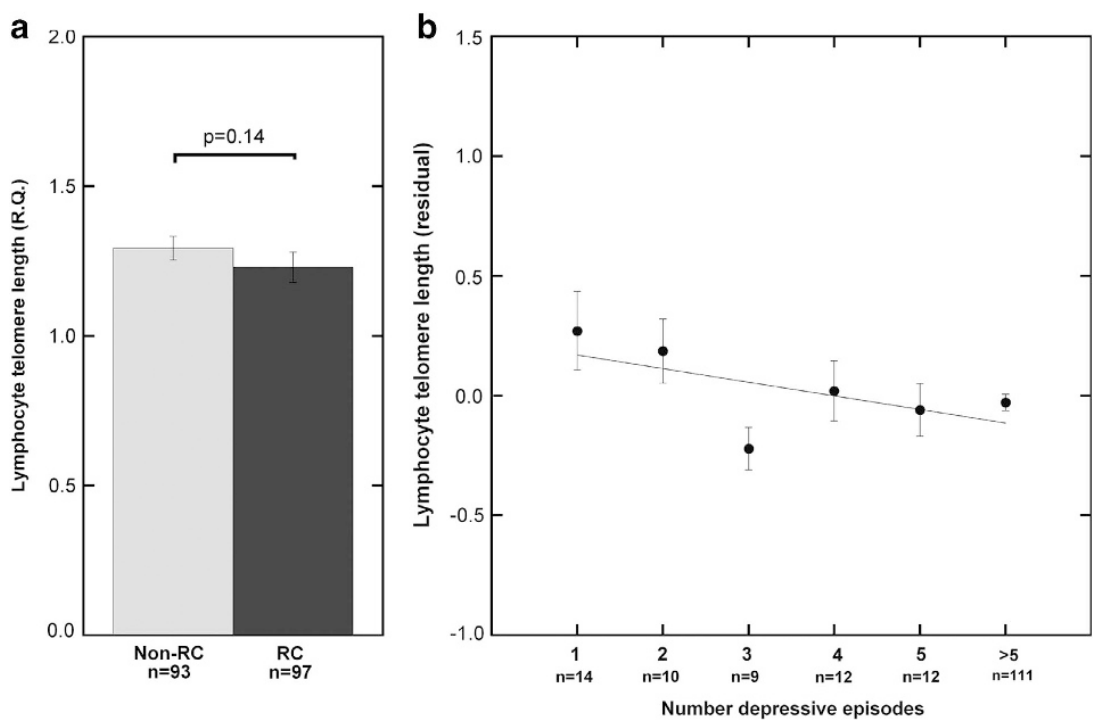

Figure 2 Lymphocyte telomere length (LTL) point estimate was reduced in those with rapid cycling $(R C ; P=0.14)(a)$, and LTL was significantly reduced with increasing number of depressive episodes $(P=0.007)(\mathbf{b})$, with larger effect in males $(P=0.006)$. The scatter plot data shown are from males and females. In $(\mathbf{a})$, the $y$ axis represents $\mathrm{LTL}$ relative quantity $(\mathrm{RQ})$ values and patients in $\mathrm{RC}$ and non-RC groups are matched for age and sex. $\ln (\mathbf{b})$, the yaxis represents unstandardized residuals of regressing $\mathrm{LTL}$ $(\mathrm{RQ})$ on age and sex. Bars and dots indicate the mean and error bars indicate standard error of the mean. 
but also non-LiR individuals $(n=60)$ had longer LTL compared with controls $(F=37.8, P<0.0005$, ANCOVA adjusted for sex $(P=0.096)$ and age $(P=0.035) ; P<0.0005$ Mann-Whitney $U$-tests). Furthermore, the patients on lithium monotherapy ( $n=39$; 53.8\% males; age 55 (39, 63), 33-73 years) had a mean LTL value that was $34.7 \%$ higher compared with controls $(\mathrm{F}=26.7, \quad P<0.0005$, partial $\eta^{2}=0.14$, ANCOVA adjusted for sex $(P=0.080)$ and age $(P=0.008) ; P<0.0005$ Mann-Whitney $U$-tests; Figure 3).

\section{Discussion}

This is the first study to report an association between lithium treatment in BD patients and LTL. BD patients on lithium had markedly longer LTL telomeres compared with healthy controls. This latter result remained significant, and equally strong, when LTL was assessed in BD patients on lithium monotherapy. The findings also suggest that duration of lithium treatment (more than 30 months) in $\mathrm{BD}$ patients correlate positively with LTL. Further, there was a tendency for $B D$ patients responding well to lithium to have longer $L T L$ than that poor responders had. Conversely, the number of depressive episodes in BD patients was associated with shorter LTL, with a stronger effect in males. This is in line with the only previous study of TL in BD, which found that BD type 2 and number of depressive episodes were associated with shorter LTL. ${ }^{20}$ However, the latter study did not investigate drug response on LTL, as only 2 of the 28 patients were on mood stabilizers (lithium or valproate). The effect sizes of the findings were moderate to large (partial $\eta^{2}$ and $R^{2} \geqslant 0.13$ ).

With regard to the lithium findings, one should bear in mind that (i) there are no lithium receptors, and (ii) the small $\mathrm{Li}^{+}$ ions enter all cells, replace sodium ions, compete with magnesium binding, and have a variety of inhibitory and/or facilitatory effects on enzymes and signaling systems. A wealth of findings has documented effects of lithium on monoaminergic systems, neurotrophic factors, neuropeptides and the arachidonic acid cascade. Lithium has also been found to enhance neuroprotective proteins and pathways (such as Bcl-2 and the Wnt signaling pathway), and to inhibit the key enzymes glycogen synthase kinase-3 (GSK-3, isoforms $\alpha$ and $\beta$ ) and phosphatidylinositol phosphatases, either directly or indirectly by affecting protein complexes that can modify the Akt/GSK-3 signaling. ${ }^{27-30}$ Besides the wide usage of lithium in both phases of BD and in major depressive disorder, investigations from the past decade have also focused on the drug's effects on a number of central nervous system disorders, including Alzheimer's disease, multiple sclerosis, amyotrophic lateral sclerosis, Huntington's disease and stroke (glutamate neurotoxicity). ${ }^{8}$ The widening of lithium's therapeutic perspective has emerged as a consequence of experiments showing that lithium is neuroprotective and anti-inflammatory, and that it also affects cell proliferation/neurogenesis, neuronal plasticity and hippocampal volume. ${ }^{31-34}$

With regard to a specific effect of lithium on TL, mechanistic support for the association between lithium treatment and LTL was recently provided. ${ }^{35}$ hTERT, a catalytic subunit bearing the enzymatic activity of telomerase, is the rate-limiting determinant of human telomerase activity, whereas the other subunits are constitutively expressed. ${ }^{36}$ In general, hTERT is considered to be regulated primarily on the transcriptional level. Specifically, transcription of $h T E R T$ has been found to be activated by $\beta$-catenin, which binds to and removes repressor T-cell factor 4 (TCF4) from the $h T E R T$ promoter, resulting in increased telomerase activity and longer $T L$ in human cancer cell lines and human embryonic kidney cells. ${ }^{35}$ $\beta$-Catenin is indirectly regulated by lithium with the main mediator between lithium and $\beta$-catenin being GSK-3 $\beta$. $\beta$-Catenin is phosphorylated by GSK-3 $\beta$, leading to $\beta$-catenin degradation. This elimination of $\beta$-catenin prevents it from reaching the nucleus for removing TCF4. Lithium inhibits GSK-3 $\beta$ by several mechanisms ${ }^{33}$ leading to retention of $\beta$-catenin that can activate $h T E R T$ transcription. ${ }^{33}$ GSK-3 $\beta$, $\beta$-catenin and hTERT are expressed in lymphocytes as well as in the brain. ${ }^{35,37,38}$ GSK-3 $\beta$ is a kinase abundantly expressed in the brain regulated by numerous signaling pathways, thus activate hTERT transcription. Lithium inhibits GSK-3 $\beta$ directly and reversibly, by regulating its transcription and by competing with $\mathrm{Mg}^{2+}$ and thereby disrupting the catalytic function of GSK-3 $\beta .^{39}$ Lithium also indirectly regulates GSK-3 $\beta$ activity by inducing various kinases and phosphatases, such as PI3K, PP2A, PKC and Akt, controlling phosphorylation at GSK-3 $\beta$ serine-9, which keeps GSK-3 $\beta$ inactive. The downstream molecular targets of GSK-3 $\beta$ are many, and the resulting functional consequences of GSK-3 $\beta$ inhibition depend on the target. Inhibition of GSK-3 $\beta$ is proposed to be one of the two core mediating events of lithium's neurotrophic and neuroprotective effects including increased neurogenesis, axonal elongation, dendrite growth, as well as reduced apoptosis and neurodegeneration. ${ }^{40}$

The second core mediating event of lithium's neuroprotective effect is the inactivation of phosphatidylinositol phosphatases. ${ }^{40}$ This inactivation blocks the recycling of inositol, which is essential for $\mathrm{IP}_{3}$-mediated signaling from G-coupled receptors and tyrosine receptors. Furthermore, this lithium-induced inositol depletion increases intracellular degradation of dysfunctional proteins or cellular components (autophagy) and stabilizes the neuronal membrane through downregulation of Myristoylated Alanine-Rich Protein Kinase C Substrate. ${ }^{41}$ Thus, in the form of downstream events, lithium influences a variety of biochemical systems that are involved in neuronal survival and differentiation. ${ }^{40,42,43}$ Because of the numerous downstream molecular targets of GSK-3 $\beta$, the abundance of $h T E R T$ regulators and the plethora of enzymes dependent on $\mathrm{Mg}^{2+}$ with which $\mathrm{Li}^{+}$may compete, there are probably several links between lithium and TL yet to be discovered.

In this study, the association of lithium with LTL was seen only after long-term treatment at a therapeutic dose (serum concentration of $0.5-0.9 \mathrm{mmoll}^{-1}$ ). Clinical empirical data have shown that it usually takes $6-12$ months of therapeutic lithium dose to reach maximum prophylactic effect in $\mathrm{BD} .^{3}$ The temporal association of lithium with LTL might be related to the clinical response to treatment.

An attempt to score the clinical response to lithium treatment has been provided through the Alda-Scale, where high score indicates LiR, that is, marked reduction in yearly number of episodes. ${ }^{7}$ Those with good response to lithium treatment had longer LTL than those with poor response. The 
LiR-LTL association did not appear to be confounded by the longer duration of lithium treatment in the LiR group compared with the non-LiR patients. Instead it may be due to the higher number of depressive episodes among non-LiR than among $\mathrm{LiR}(P<0.0005$, Mann-Whitney $U$-tests). Also, we cannot rule out the possibility of different lithium concentrations in the serum of LiR and non-LiR groups, although such a difference would be unexpected due to the treatment routines that are followed.

Previous studies have linked stress and psychiatric disorders such as schizophrenia and depression to shorter telomeres. ${ }^{12,16,17,19}$ Accordingly, in this sample an increased number of depressive episodes was associated with shorter $L T L$ in the BD patients. Thus, we hypothesize that LTL in BD patients will reflect a balance between the number of depressive episodes and the duration of treatment with therapeutic lithium concentrations. Remarkably, the LTL was markedly longer in BD patients compared with healthy controls. The LTL was longer than the controls in both the LiR group and the non-LiR group. Previously, shorter LTL in schizophrenia was associated with poor response to antipsychotic treatment, ${ }^{19}$ and higher telomerase activity was associated with antidepressant pharmacological treatment in unipolar depression. ${ }^{19}$ Similarly, lithium might influence the telomerase activity and contribute to longer LTL. Therefore, we studied BD patients on lithium monotherapy as a separate subgroup and the results showed that the lithium treatment association with LTL was not confounded by other drugs. There is, to our knowledge, no previous report on LTL with regard to manic/ hypomanic episodes. In this study, no association was found between LTL and number of manic or hypomanic episodes. On the other hand, TL has repeatedly been found to be inversely correlated with increased age. Accordingly, we found that LTL decreased with increased age both in patients and controls. Consequently, age was controlled for in the statistical analyses. Similarly, we also controlled for sex and the association between LTL and number of depressive episodes was found to be stronger in males.

The intriguing possibility that a decreased length of telomeres could be a marker of BD disease, based on that a wide variety of insults and pathologies reduce TL, cannot be answered by our data as almost all BD patients were treated with lithium, well established as mood stabilizer in Sweden. ${ }^{44}$ In this study, BD patients had markedly longer telomeres than the healthy controls. Our heuristic hypothesis is that the effects of lithium treatment were overriding the impact of negative events. In order to clarify this issue, samples from a cohort of not previously treated BD patients are needed, which is part of our current project planning. Other weaknesses in this study include: (i) The study design was cross-sectional/ retrospective yielding data that were only associative in nature. Investigations of the putative causality between lithium and LTL should be pursued in the future. (ii) Shorter LTL has been reported to be associated with cigarette smoking, obesity, inflammation and several somatic diseases. ${ }^{11,34}$ These factors were not controlled for in the present study as these data were not available. Nonetheless, there is no clinical indication of less nicotine use or lower body weight in $\mathrm{BD}$ patients compared with the general population in Stockholm, Sweden, where the samples derived from; (iii) Almost all Swedish BD patients have received other pharmacological treatments before lithium because of the well-known delay in correct BD diagnosis. Therefore, it was not possible to study the effect of each drug separately before the start of lithium treatment using the current study design. Instead, BD patients on lithium monotherapy were studied as a subgroup, which supported that the data showing longer LTL after lithium treatment were not a consequence of therapy with other drugs. (iv) The number of depressive episodes and the number of manic or hypomanic episodes larger than six was indicated as ' 6 ' implying loss of information. However, the LTL residuals, after correction for age and sex for those with more than five depressive episodes, displayed a narrow 95\% confidence interval at LTL values higher than the point estimate of the five episodes group. (v) Similar to previous TL studies of psychiatric disorders and stress, TL was here measured in a peripheral tissue and not in the brain where lithium is considered to exert its therapeutic action. (vi) For the test of association for LTL to RC and number of depressive episodes in sample Set II, a significance threshold of $P<0.025$ may be appropriate as LTL was studied for dependence also on number of manic/hypomanic episodes in this set. The finding that LTL was negatively associated with number of depressive episodes $(P=0.007)$ survived the more stringent significance threshold, and was also confirmed using Set I.

Clinical implications. Our findings add significantly to knowledge regarding the putative mechanisms of lithium action. Specifically, the results showed markedly longer LTL in BD patients treated with lithium, suggesting a lithium-induced increase of telomerase activity during lymphopoiesis. A similar lithium effect during neurogenesis would be in line with previously proposed neuroprotective effects of lithium, as telomerase activity and TL influence neural stem cell proliferation and neurogenesis in neurogenic regions of the adult brain. ${ }^{45}$ Importantly, the LTL was increased in BD patients responding well to lithium compared with non-responders. Lithium treatment is the first line of maintenance treatment in BD but a significant number of patients are only partial responders and a significant proportion of patients require add-on pharmacological treatment with antiepileptics and antipsychotics in order to prevent relapses. Although a distinct family history of bipolarity, typical clinical picture and the maniadepression-remission sequence of disease are good clinical LiR predictors, no biomarkers of response have been identified so far. Identification of a response trait marker would alleviate a significant unmet medical need as it would enable a priori choice of optimal individual maintenance treatment. Furthermore, identification of such a marker could guide additional investigations of lithium's therapeutic role in neurodegenerative and other disorders. ${ }^{8}$ Finally, it remains to be tested if agents influencing TL might be useful as augmentation agents in treatment of intermediate lithium responders.

\section{Conclusions}

These data suggest that long-term lithium treatment in BD patients results in longer LTL with an effect size that is stronger than the telomere shortening effect of depressive 
episodes. The association between lithium and LTL is supported by a previously reported mechanistic pathway linking lithium and telomerase in vitro. We propose prospective studies of telomerase activity and $T L$ in lithium-treated BD patients in order to elucidate LTL's role in $\mathrm{BD}$ and in the therapeutic effects of lithium.

\section{Conflict of interest}

LM has taken part in a conference on bipolar disorder arranged by Bristol-Myers Squibb. The remaining authors declare no conflict of interest.

Acknowledgements. This project was supported by grants from Karolinska Institutet, the Swedish Medical Research Council, The SöderströmKönigska Foundation, the Lundbeck Foundation, The Bror Gadelius Foundation and Psychiatry Southwest, Stockholm. Financial support was also provided through the regional agreement on medical training and clinical research (ALF) between the Stockholm County Council and Karolinska Institutet. We want to thank all the patients who participated in this study. We also thank Inger Römer-Ek, RN, MSc, for skillful assistance.

1. Bagalman E, Muser E, Choi JC, Durden E, Macfadden W, Haskins JT et al. Health care resource utilization and costs in a commercially insured population of patients with bipolar disorder type I and frequent psychiatric interventions. Clin Ther 2011; 33: 1381-1390 e4.

2. Osby U, Tiainen A, Backlund L, Edman G, Adler M, Hallgren J et al. Psychiatric admissions and hospitalization costs in bipolar disorder in Sweden. J Affect Disord 2009; 115: 315-322.

3. Goodwin FK, Jamison KR. Manic Depressive Illness: bipolar disorders and recurrent depression. Oxford University Press: Oxford, 2007.

4. Bauer M, Beaulieu S, Dunner DL, Lafer B, Kupka R. Rapid cycling bipolar disorderdiagnostic concepts. Bipolar Disord 2008; 10(1 Pt 2): 153-162.

5. Baldessarini RJ, Tondo L, Hennen J. Treating the suicidal patient with bipolar disorder. Reducing suicide risk with lithium. Ann N Y Acad Sci 2001; 932: 24-38; discussion 39-43.

6. Licht RW. Lithium: still a major option in the management of bipolar disorder. CNS Neurosci Ther 2012; 18: 219-226.

7. Grof $P$, Duffy A, Cavazzoni P, Grof E, Garnham J, MacDougall M et al. Is response to prophylactic lithium a familial trait? J Clin Psychiatry 2002; 63: 942-947.

8. Chiu CT, Chuang DM. Neuroprotective action of lithium in disorders of the central nervous system. Zhong Nan Da Xue Xue Bao Yi Xue Ban 2011; 36: 461-476.

9. Zanni GR, Wick JY. Telomeres: unlocking the mystery of cell division and aging. Consult Pharm 2011; 26: 78-90.

10. Barrett EL, Richardson DS. Sex differences in telomeres and lifespan. Aging Cell 2011; 10: 913-921.

11. Babizhayev MA, Savel'yeva EL, Moskvina SN, Yegorov YE. Telomere length is a biomarker of cumulative oxidative stress, biologic age, and an independent predictor of survival and therapeutic treatment requirement associated with smoking behavior. Am J Ther 2011; 18: e209-e226.

12. Wolkowitz OM, Reus VI, Mellon SH. Of sound mind and body: depression, disease, and accelerated aging. Dialogues Clin Neurosci 2011; 13: 25-39.

13. Wikgren M, Maripuu M, Karlsson T, Nordfjall K, Bergdahl J, Hultdin J et al. Short telomeres in depression and the general population are associated with a hypocortisolemic state. Biol Psychiatry 2012; 71: 294-300.

14. Ludlow AT, Zimmerman JB, Witkowski S, Hearn JW, Hatfield BD, Roth SM. Relationship between physical activity level, telomere length, and telomerase activity. Med Sci Sports Exerc 2008; 40: 1764-1771.

15. Wolkowitz OM, Mellon SH, Epel ES, Lin J, Reus VI, Rosser R et al. Resting leukocyte telomerase activity is elevated in major depression and predicts treatment response. $\mathrm{Mo}$ Psychiatry 2012; 17: 164-172.

16. Okereke OI, Prescott J, Wong JY, Han J, Rexrode KM, De Vivo I. High phobic anxiety is related to lower leukocyte telomere length in women. PLOS One 2012; 7: e40516.

17. Wolkowitz OM, Mellon SH, Epel ES, Lin J, Dhabhar FS, Su Y et al. Leukocyte telomere length in major depression: correlations with chronicity, inflammation and oxidative stress-preliminary findings. PLoS One 2011; 6: e17837.

18. Simon NM, Smoller JW, McNamara KL, Maser RS, Zalta AK, Pollack MH et al. Telomere shortening and mood disorders: preliminary support for a chronic stress model of accelerated aging. Biol Psychiatry 2006; 60: 432-435.

19. Yu WY, Chang HW, Lin CH, Cho CL. Short telomeres in patients with chronic schizophrenia who show a poor response to treatment. J Psychiatry Neurosci 2008; 33: 244-247.

20. Elvsashagen T, Vera E, Boen E, Bratlie J, Andreassen OA, Josefsen D et al. The load of short telomeres is increased and associated with lifetime number of depressive episodes in bipolar II disorder. J Affect Disord 2011; 135: 43-50.
21. Backlund L, Lavebratt $C$, Frisen L, Nikamo $P$, Hukic Sudic D, Traskman-Bendz $L$ et al. P2RX7: expression responds to sleep deprivation and associates with rapid cycling in bipolar disorder type 1. PLoS One 2012; 7: e43057.

22. Schulze TG, Alda M, Adli M, Akula N, Ardau R, Bui ET et al. The International Consortium on Lithium Genetics (ConLiGen): an initiative by the NIMH and IGSLI to study the genetic basis of response to lithium treatment. Neuropsychobiology 2010; 62: 72-78.

23. Almgren M, Atkinson R, He J, Hilding A, Hagman E, Wolk A et al. Adenovirus-36 is associated with obesity in children and adults in sweden as determined by rapid ELISA. PLoS One 2012; 7: e41652.

24. Sjoholm L, Lavebratt C, Forsell Y. A multifactorial developmental model for the etiology of major depression in a population-based sample. J Affect Disord 2009; 113: 66-76.

25. Cawthon RM. Telomere measurement by quantitative PCR. Nucleic Acids Res 2002; 30 : e47.

26. Kananen L, Surakka I, Pirkola S, Suvisaari J, Lonnqvist J, Peltonen L et al. Childhood adversities are associated with shorter telomere length at adult age both in individuals with an anxiety disorder and controls. PLoS One 2010; 5: e10826.

27. Angelucci $F$, Aloe L, Jimenez-Vasquez $P$, Mathe AA. Lithium treatment alters brain concentrations of nerve growth factor, brain-derived neurotrophic factor and glial cell linederived neurotrophic factor in a rat model of depression. Int $J$ Neuropsychopharmacol 2003; 6: 225-231.

28. Husum $H$, Mathe AA. Early life stress changes concentrations of neuropeptide $Y$ and corticotropin-releasing hormone in adult rat brain. Lithium treatment modifies these changes. Neuropsychopharmacology 2002; 27: 756-764.

29. Johnson L, El-Khoury A, Aberg-Wistedt A, Stain-Malmgren R, Mathe AA. Tryptophan depletion in lithium-stabilized patients with affective disorder. Int $J$ Neuropsychopharmacol 2001; 4: 329-336.

30. Miller JC, Jimenez P, Mathe AA. Restraint stress influences AP-1 and CREB DNA-binding activity induced by chronic lithium treatment in the rat frontal cortex and hippocampus. Int $J$ Neuropsychopharmacol 2007; 10: 609-619.

31. van Erp TG, Thompson PM, Kieseppa T, Bearden CE, Marino AC, Hoftman GD et al. Hippocampal morphology in lithium and non-lithium-treated bipolar I disorder patients, nonbipolar co-twins, and control twins. Hum Brain Mapp 2012; 33: 501-510.

32. Li H, Li Q, Du X, Sun Y, Wang X, Kroemer G et al. Lithium-mediated long-term neuroprotection in neonatal rat hypoxia-ischemia is associated with antiinflammatory effects and enhanced proliferation and survival of neural stem/progenitor cells. J Cereb Blood Flow Metab 2011; 31: 2106-2115.

33. Chuang DM, Wang Z, Chiu CT. GSK-3 as a target for lithium-induced neuroprotection against excitotoxicity in neuronal cultures and animal models of ischemic stroke. Front $\mathrm{Mol}$ Neurosci 2011; 4: 15

34. Forlenza OV, de Paula VJ, Machado-Vieira R, Diniz BS, Gattaz WF. Does lithium prevent Alzheimer's disease? Drugs Aging 2012; 29: 335-342.

35. Zhang $Y$, Toh L, Lau P, Wang X. Human telomerase reverse transcriptase (hTERT) is a novel target of the Wnt/beta-catenin pathway in human cancer. J Biol Chem 2012; 287: 32494-32511.

36. Hartwig FP, Nedel F, Collares TV, Tarquinio SB, Nor JE, Demarco FF. Telomeres and tissue engineering: the potential roles of TERT in VEGF-mediated angiogenesis. Stem Cell Rev 2012; 8: 1275-1281.

37. Lovatt M, Bijlmakers MJ. Stabilisation of beta-catenin downstream of $\mathrm{T}$ cell receptor signalling. PLoS One 2010; 5: 9.

38. Liu C, Kato Y, Zhang Z, Do VM, Yankner BA, He X. beta-Trcp couples beta-catenin phosphorylation-degradation and regulates Xenopus axis formation. Proc Natl Acad Sci USA 1999; 96: 6273-6278.

39. Klein PS, Melton DA. A molecular mechanism for the effect of lithium on development. Proc Natl Acad Sci USA 1996; 93: 8455-8459.

40. Pasquali L, Busceti CL, Fulceri F, Paparelli A, Fornai F. Intracellular pathways underlying the effects of lithium. Behav Pharmacol 2010; 21: 473-492.

41. Sarkar S, Floto RA, Berger Z, Imarisio S, Cordenier A, Pasco M et al. Lithium induces autophagy by inhibiting inositol monophosphatase. J Cell Biol 2005; 170: 1101-1111.

42. Chiu CT, Chuang DM. Molecular actions and therapeutic potential of lithium in preclinical and clinical studies of CNS disorders. Pharmacol Ther 2010; 128: 281-304.

43. Machado-Vieira R, Manji HK, Zarate Jr CA. The role of lithium in the treatment of bipolar disorder: convergent evidence for neurotrophic effects as a unifying hypothesis. Bipolar Disord 2009; 11(Suppl 2): 92-109.

44. Backlund L, Ehnvall A, Hetta J, Isacsson G, Agren H. Identifying predictors for good lithium response-a retrospective analysis of 100 patients with bipolar disorder using a lifecharting method. Eur Psychiatry 2009; 24: 171-177.

45. Lee SW, Clemenson GD, Gage FH. New neurons in an aged brain. Behav Brain Res 2012; 227: 497-507.

(c) (i)(2)(2) Translational Psychiatry is an open-access journal By ${ }_{\mathrm{B}} \mathrm{SA}$ published by Nature Publishing Group. This work is licensed under a Creative Commons Attribution-NonCommercialShareAlike 3.0 Unported License. To view a copy of this license, visit http://creativecommons.org/licenses/by-nc-sa/3.0/ 